\title{
Echographie Pédiatrique En Pratique Hospitaliere Au Centre Hospitalier Universitaire (CHU) De Kara Au Togo
}

\author{
B. N'timon \\ Service De Radiologie Et Imagerie Médicale CHU Kara, Togo \\ P. Gbande
}

Service De Radiologie Et Imagerie Médicale CHU de Lomé, Togo

M. Dagbe

Service De Radiologie Et Imagerie Médicale CHU Kara, Togo

T. Dossouvi

Service De Chirurgie Générale CHU Kara, Togo

M. Tchaou

A. Amadou

L. Sonhaye

L.K. Agoda-Koussema

K.V. Adjenou

Service De Radiologie Et Imagerie Médicale CHU de Lomé, Togo

Doi: 10.19044/esj.2018.v14n6p443 URL:http://dx.doi.org/10.19044/esj.2018.v14n6p443

\begin{abstract}
Purpose: To list pediatric ultrasound exams performed at Kara University Hospital Center in Togo and the pathologies observed. Materials and methods: This was a retrospective and descriptive study of the results of pediatric ultrasound examinations performed in the Department of Radiology and Imaging of Kara University Hospital Center. The study ran from January 1, 2013 to December 31, 2016, over a period of 3 years. Results: The frequency of pediatric ultrasounds was $5.1 \%$. The average age of the patients was 2.16 years with extremes of 1 day to 15 years. There was a male predominance with a sex ratio of 1.4. Abdomino-pelvic ultrasound were the most represented, $92 \%$, followed by cervical ultrasound in $2.6 \%$ of cases. Abdominal ultrasound indications were dominated by abdominal pain in $33.6 \%$ of cases, followed by palpation of abdominal mass in $15.7 \%$. Cervical ultrasounds were motivated in 5 out of 8 cases by the cervical swelling. Abdomino-pelvic ultrasonography was pathological in $70.1 \%$ of cases. Hepatic affections were the most common abdominal abnormality in $37.8 \%$ of cases followed by splenic disorders. Hepatic lesions were dominated by
\end{abstract}


homogeneous hepatomegaly with $63.2 \%$ of cases. Cervical ultrasound was pathological in 6 out of 8 cases. The lesions observed were mainly cervical lymphadenopathy (4 cases out of 6). Conclusion: Ultrasound is a very useful tool in the exploration of pediatric diseases. The abdominal ultrasound is the most performed and hepatic pathologies are the most common.

Keywords: Ultrasound, child, pediatrics, abdomen, cervical, Togo

\section{Résumé}

But : Répertorier les examens échographiques pédiatriques réalisés et les lésions observées dans le service de radiologie du CHU Kara au Togo. Matériels et méthodes: Il s'est agi d'une étude rétrospective et descriptive portant sur les résultats des examens d'échographies pédiatriques réalisés dans le service de Radiologie et Imagerie Médicale du CHU Kara. L'étude s'est déroulée du $1^{\text {er }}$ janvier 2013 au 31 décembre 2016 soit sur une période de 3 ans. Résultats : la fréquence des échographies pédiatriques était de 5,1\%. L'âge moyen des patients était de 2,16 ans avec des extrêmes de 1 jour à 15 ans. On notait une prédominance masculine avec une sex-ratio de 1,4. Les échographies abdomino-pelviennes étaient les plus représentées (92\%), suivi des échographies cervicales dans $2,6 \%$ des cas. Les indications des échographies abdomino-pelviennes étaient dominées par les douleurs abdominales dans $33,6 \%$ des cas, suivi de la palpation d'une masse abdominale dans $15,7 \%$. Les échographies cervicales étaient motivées dans 5 cas sur 8 par une tuméfaction cervicale. Les échographies abdominopelviennes étaient pathologiques dans $70,1 \%$ des cas. Les affections hépatiques étaient l'anomalie abdominale la plus représentée (37,8\% des cas) suivi des affections spléniques. Les lésions hépatiques étaient dominées par l'hépatomégalie homogène avec $63,2 \%$ des cas. L'échographie cervicale était pathologique dans 6 cas sur 8 . Les lésions observées étaient principalement les adénopathies cervicales (4 cas sur 6). Conclusion : l'échographie est un outil très utile dans l'exploration des affections pédiatriques. L'échographie abdominale est la plus réalisée et les pathologies hépatiques sont les plus fréquentes.

Mots-clés : Échographie, enfant, pédiatrie, abdomen, cervicale, Togo

\section{Introduction}

La radiopédiatrie ou imagerie pédiatrique est la radiologie pratiquée chez les enfants. Elle constitue aujourd'hui une surspécialisation et fait appelle à plusieurs modalités d'imagerie dont l'échographie. L'échographie se définie comme est une technique d'imagerie médicale qui explore un organe ou une région du corps au moyen des corps au moyen des ultrasons (Delamare, 2006). 
Du fait de son innocuité, son accessibilité et son coût relativement abordable, l'échographie se présente comme l'examen d'imagerie médicale par excellence pour l'exploration de la pathologie pédiatrique à cause du faible panicule adipeux observée chez ces enfants (Erdemir, et al., 2013). Elle doit être privilégiée surtout ces chez les enfants qui sont des êtres en croissance et dont les tissus sont très radiosensibles (Chateil, Rouby, Brun, Labessan, \& Diard, 2004), car elle est non irradiante.

L'échographie est la modalité d'imagerie médicale la plus disponible en Afrique, et son accessibilité ne cesse de s'accroitre même dans les milieux reculés. Une étude réalisée en 2016 à Lomé, a montré que l'échographie abdomino-pelvienne chez l'enfant représentait $2,3 \%$ de toutes les échographies réalisées (Tchaou, et al., 2017). Le manque de données sur la pratique de l'échographie pédiatrique en général a motivé le choix de ce sujet. Ce travail a pour objectif général de répertorier les différents examens échographiques pédiatriques réalisés au CHU Kara et de façon plus spécifique étudier leur fréquence, leurs indications, et les principales lésions observées selon les organes.

\section{Matériels et méthodes}

Il s'est agi d'une étude monocentrique, rétrospective et descriptive portant sur les résultats des examens d'échographies pédiatriques réalisés dans le service de radiologie et imagerie médicale du CHU Kara. Le CHU Kara est le centre de référence national de la partie septentrionale du Togo. L'étude s'est déroulée du 1er janvier 2013 au 31 décembre 2016 soit sur une période de 3 ans. Nous avons inclus dans cette étude tous les résultats des examens d'échographie des enfants de 0 à 15 ans réalisés durant la période d'étude. Les données ont été collectées à partir une fiche d'enquête préétablie élaborée à cet effet. Les paramètres étudiés étaient : l'âge, le sexe, le type d'échographie, l'indication de l'échographie et les résultats de l'examen. Les appareils d'échographie utilisés étaient de marque LOGIQ 200 de GE Healthcare et ATL 5000 de Philips. Les données recueillies ont été traitées par le logiciel EPI info 7.

\section{Résultats}

Nous avons réalisé 6142 examens échographiques pendant la période d'étude dont 311 échographies pédiatriques soit une fréquence de 5,1\% de l'ensemble des échographies réalisées, avec une moyenne de 103 échographies pédiatriques par an. L'âge moyen des patients était de 2,16 ans avec des extrêmes de 1 jour à 15 ans. On notait une prédominance masculine avec une sex-ratio de 1,4. La tranche d'âge la plus représentée est celle de 12 à 15 ans (30,5\%) (Figure 1). 


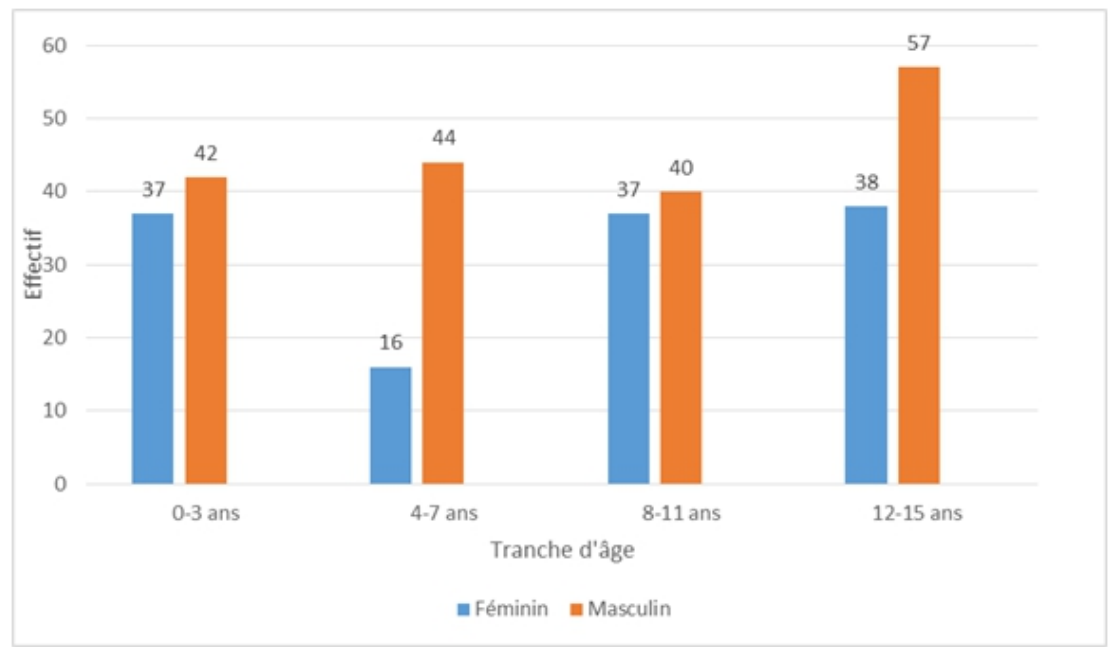

Figure 1 : Répartition des patients en fonction de l'âge et du sexe

\section{Les types d'échographie}

Les types d'échographie sont présentés dans le tableau I. Les échographies abdomino-pelviennes étaient les plus représentées (92\%) correspondant pour les deux à une fréquence de $4,7 \%$.

Tableau I : Répartition des patients selon le type d'échographie

\begin{tabular}{ccc}
\hline & $\begin{array}{c}\text { Fréquence } \\
(\mathrm{N})\end{array}$ & $\begin{array}{c}\text { Pourcentage } \\
(\%)\end{array}$ \\
\hline Abdominale -pelvienne & 286 & 92 \\
Cervicale & 8 & 2,6 \\
Parties molles & 5 & 1,6 \\
Transfontanellaire & 5 & 1,6 \\
Bourses & 5 & 1,6 \\
Mammaire & 1 & 0,3 \\
Oculaire & 1 & 0,3 \\
Total & $\mathbf{3 1 1}$ & $\mathbf{1 0 0}$ \\
\hline
\end{tabular}

\section{Les indications}

Les indications des échographies abdomino-pelviennes sont présentées dans le tableau II. Elles étaient dominées par la douleur abdominale dans $33,6 \%$ des cas.

Les échographies cervicales étaient motivées dans 3 cas sur 8 par la suspicion d'adénopathies et dans les autres cas par une tuméfaction cervicale. Les indications de l'échographie transfontanellaire (ETF) étaient : la macrocrânie ( 4 cas sur 5) et le spina bifida (1 cas). La grosse bourse était l'indication de toutes les échographies des bourses. 
Tableau II : Répartition des échographies abdomino-pelviennes selon les indications

\begin{tabular}{ccc}
\hline & Fréquence & $\begin{array}{c}\text { Pourcentage } \\
\%\end{array}$ \\
\hline Douleur abdominale & & 33,6 \\
Masse abdominale & 96 & 15,7 \\
Ballonnement abdominal & 45 & 9,8 \\
Syndrome odémato-ascitique & 28 & 5,6 \\
Hépatopathie & 16 & 12,2 \\
Contusion abdominale & 35 & 5,2 \\
Splénomégalie & 15 & 7,3 \\
Vomissements & 21 & 3,5 \\
Ectopie testiculaire & 10 & 2,4 \\
Insuffisance rénale & 7 & 2,1 \\
Autres* & 6 & 2,4 \\
\hline
\end{tabular}

*(anémie $=2 ;$ diabète $=1 ;$ aménorrhée $=2 ;$ ménarche précoce $=1 ;$ pancytopénie $=1$ )

\section{Les résultats observés lors des différentes échographies.}

Les échographies abdomino-pelviennes étaient normales chez 85 enfants soit $29,7 \%$. Elles présentaient au moins une anomalie dans les autres cas $(n=201$, soit 70,1\%). Les affections hépatiques étaient les plus représentées $(\mathrm{n}=76$ soit $37,8 \%)$ suivi des affections spléniques $(\mathrm{n}=47$ soit $16,4 \%)$ et rénales $(n=35$ soit $12,2 \%)$. On notait 23 affections digestives soit $8 \%$ des cas.

L'ascite était retrouvée chez 17 patients soit 5,9\% et les adénopathies profondes étaient présentes dans 12 cas soit 4,2\%. Les principales lésions abdomino-pelviennes sont résumées dans le tableau III. Les lésions hépatiques étaient dominées par l'hépatomégalie homogène, avec 48 cas soit $63,2 \%$. On notait dans ce groupe 4 cas de foie cardiaque. La splénomégalie homogène représentait 29 cas des lésions spléniques soit $61,7 \%$. La souffrance rénale était la principale lésion rénale observée $(\mathrm{n}=21$ soit $60 \%)$. L'aérocolie diffuse était la plus observée dans les anomalies digestives $(n=18$ soit 78,3\%).

L'appendicite aiguë et l'invagination intestinale aiguë (figure 2) ont été retrouvées chacune chez 2 patients soit $8,7 \%$. La sténose hypertrophique du pylore (figure 3) chez un patient. La cholécystite aigue a était la plus fréquente des anomalies de la vésicule biliaire 5 cas sur 9, avec 3 cas alithiasiques et 2 cas sur lithiase vésiculaire. La recherche de testicule non descendu a été infructueuse dans 6 cas sur 7. 


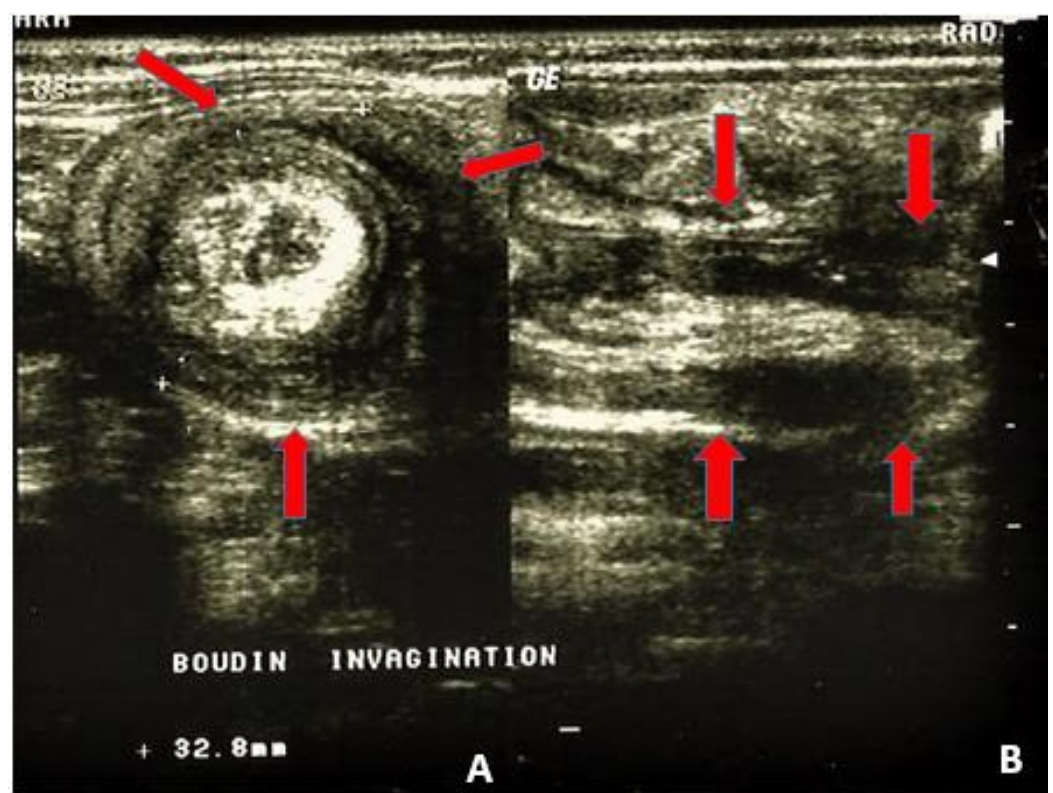

Figure 2 : Invagination intestinale aiguë (flèches rouges), image en cocarde sur la coupe transversale (A) et en sandwich sur la coupe longitudinale (B)

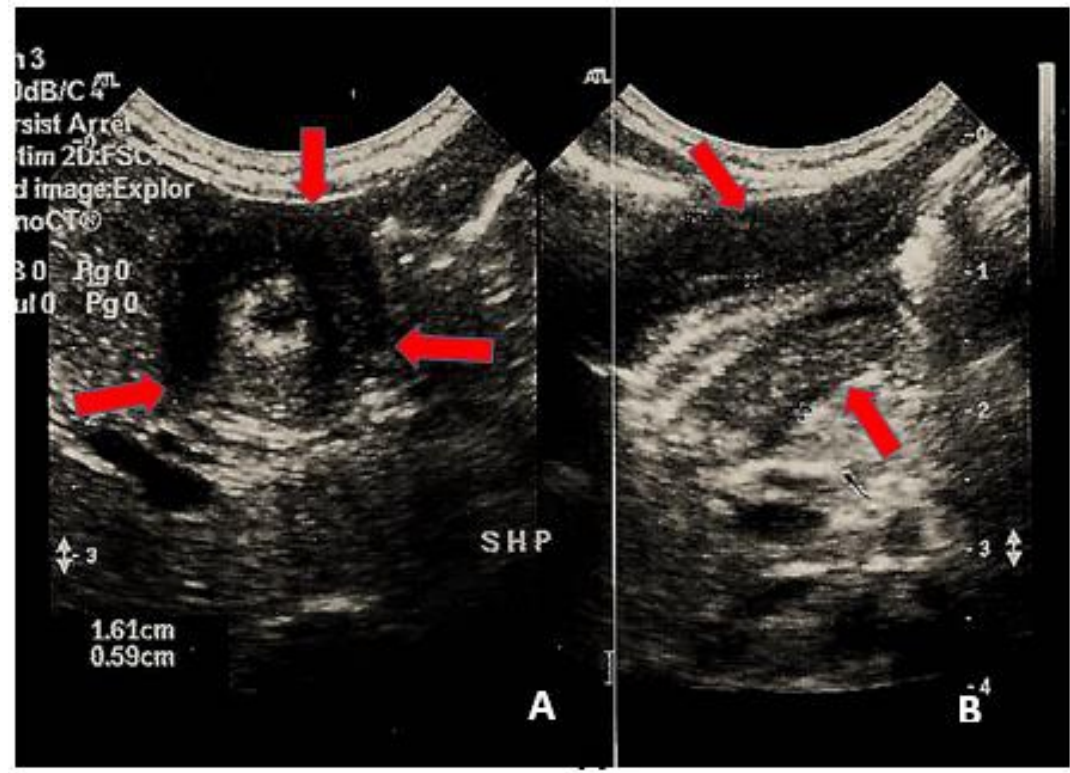

Figure 3 : Epaississement du muscle pylorique (flèches rouges) mesurant $6 \mathrm{~mm}$ d'épaisseur et $16 \mathrm{~mm}$ de long (coupe transversale $=\mathrm{A}$; coupe longitudinale $=\mathrm{B}$ ) traduisant une sténose hypertrophique du pylore avec dilatation gastrique (E)

L'échographie cervicale était pathologique dans 6 cas sur 8 . Les lésions observées étaient : les adénopathies cervicales (4 cas sur 6), 1 cas de kyste branchial et 1 cas de parotidite bilatérale abcédée. L'échographie des parties molles avait retrouvé 2 cas d'abcès sous-périostés dans le cadre de 
l'exploration des ostéomyélites, 2 cas de tératomes saccro-coccygiens et 1 cas de méningo-encéphalocèle dans le cadre d'un spina bifida. L'échographie transfontannellaire était normale dans 2 cas sur 5. Les lésions observées étaient essentiellement l'hydrocéphalie (2 cas d'hydrocéphalie triventriculaire et 1 cas tétéventriculaire). L'échographie des bourses était pathologique dans 4 cas sur 5. Les lésions observées étaient : 2 cas de kyste du cordon spermatique, un cas d'hydrocèle et 1 cas de hernie inguino-scrotale. L'échographie oculaire réalisée était normale.

Tableau III : Répartition des principales lésions abdominales observées par organe

$$
\text { n }(\%)
$$

\begin{tabular}{cc}
\hline Foie & \\
Hépatomégalie homogène & $\mathbf{7 6}(\mathbf{1 0 0})$ \\
Hépatomégalie nodulaire & $48(63,2)$ \\
Stéatose hépatique & $13(17,1)$ \\
Lésion focale & $10(13,2)$ \\
Foie multikystique & $4(5,3)$ \\
Rate $\quad 1(1,3)$ \\
Splénomégalie homogène & $\mathbf{4 7}(100)$ \\
Splénomégalie hétérogène & $29(61,7)$ \\
Lésion focale & $12(25,5)$ \\
Asplénie & $4(8,5)$ \\
Reins et voies urinaires & $2(4,3)$ \\
Souffrance rénale & $\mathbf{3 5}(100)$ \\
Gros reins multikystiques & $21(60)$ \\
Urétéro-hydronéphrose & $2(5,7)$ \\
Masse rénale & $5(14,3)$ \\
Ectopie rénale & $4(11,4)$ \\
Rein unique & $1(2,9)$ \\
Anomalie de jonction pyélo-calicielle & $1(2,9)$ \\
& $1(2,9)$ \\
\hline
\end{tabular}

\section{Discussion}

La fréquence des échographies pédiatriques réalisées pendant notre période d'étude était de 5,1\% avec une moyenne de 103 échographies pédiatriques par an et une fréquence de $4,7 \%$ pour les échographies abdomino-pelviennes qui représentaient l'essentiel des examens d'échographie $(92 \%)$. Cette fréquence est légèrement supérieure à celle de Tchaou et al qui trouvé une fréquence de 2,3\% pour les échographies abdomino- pelvienne (Tchaou, et al., 2017). L'âge moyen des patients était de 2,16 ans avec des extrêmes de 1 jour à 15 ans. Ce résultat est bien inférieur à ceux observés dans la littérature (Tchaou, et al., 2017; Mabiala-Babela, Pandzou, Koutaba, Ganga-Zandzou, \& Sanga, 2006; Kouame, et al., 2017). On notait une prédominance masculine avec une sex-ratio de 1,4. Cette 
prédominance masculine s’observe également dans plusieurs études (Tchaou, et al., 2017; Kouame, et al., 2017; Agoda-Koussema, et al., 2013).

Les échographies abdominales et abdomino-pelviennes étaient les plus représentées. Elles constituent à elles seules $92 \%$ des examens échographiques. Ceci montre que l'échographie est de loin la modalité d'imagerie la plus sollicitée pour l'exploration de l'abdomen de l'enfant. Elle est aujourd'hui plus demandée dans les abdomens aigus chez les enfants que la radiographie de l'abdomen sans préparation avec laquelle elle constitue un couple toujours d'actualité dans ces pathologies (Kouame, et al., 2017). Les indications de ces échographies abdominales dans notre étude étaient dominées par la douleur abdominale. Ce résultat est superposable à celui obtenu par Tchaou et al Tchaou, et al., 2017). La palpation d'une masse abdominale était la 2ème indication observée dans la demande de ces échographies abdominales. En effet l'échographie est l'examen de première intention devant une masse abdominale (Hanquinet, Ferey, \& Kalifa, 2009). Elle permettra de préciser la topographie de la masse (intra- ou retropéritonéale) ; l'aspect (liquide, solide ou mixte). Elle permettra aussi de rechercher la vascularisation de la masse et l'envahissement des organes voisins (en cas de tumeur maligne). En fonction des résultats de l'échographie et l'orientation diagnostique, une autre modalité d'imagerie en coupe complètera celle-ci.

Les échographies abdominales avaient révélé au moins une pathologie dans 70,1\%. Tchaou et al avaient trouvé $61,3 \%$ d'examen pathologiques (Tchaou, et al., 2017). Les affections hépatiques étaient les plus présentes suivi des affections spléniques et rénales. Parmi les lésions hépatiques, 1'hépatomégalie homogène était la plus fréquemment retrouvée dans près des 2/3 des cas. Elle était en majorité isolée et peut rentrer dans le cadre des maladies parasitaires et infectieuses dans notre milieu africain en zone tropicale, ou dans le cadre des maladies de surcharge hépatique notamment une glycogénose. L'hépatomégalie nodulaire est le plus souvent l'expression de tumeurs hépatiques. Dans notre série on notait 13 cas d'hépatomégalie nodulaire qui étaient soit une tumeur bénigne ou maligne. Les tumeurs malignes sont fréquemment des métastases. Plus rarement, il peut s'agir d'une tumeur maligne primitive [9]. Les métastases hépatiques sont rarement révélatrices d'une tumeur primitive à l'exception du syndrome de Pepper [10].

L'hépatomégalie pouvait être associée à une splénomégalie homogène qui était la principale lésion splénique observée. L’hépato-splénomégalie homogène traduit des lésions infectieuses dans nos milieux ou une maladie de surcharge. Chez le nouveau-né ou le petit nourrisson, il faut penser en plus des lésions infectieuses, à la mucolipidose de type II (I-cell-disease), la galactosialidose (forme précoce), la maladie de Landing. Chez le nourrisson plus grand et chez l'enfant, une hépatosplénomégalie isolée peut faire évoquer 
en plus des maladies infectieuses, une maladie de Nieman Pick type B ou une maladie de Gaucher de type I. La splénomégalie pouvait être isolée. Elle traduit une lésion infectieuse ou la splénomégalie des anémies hémolytiques constitutionnelles notamment la drépanocytose dans notre contexte. Elle peut être aussi le mode de révélation d'une hypertension portale (Labrune, Trioche-Eberschweiler, Mollet-Boudjemline, \& Gajdos, 2007).

La souffrance rénale ou encore néphropathie médicale était la principale lésion rénale observée. Le diagnostic sera évoqué en fonction du changement de la taille des reins. $\mathrm{Si}$ la taille des reins est conservée, on évoquera une néphrocalcinose ou une tubulopathie. Si les reins sont de grande taille, il s'agit le plus souvent d'une glomérulonéphrite aiguë, d'un syndrome néphrotique ou d'un syndrome hémolyse-urémie (Hanquinet, Ferey, \& Kalifa, 2009).

La recherche de testicule non palpée dans la bourse a motivé la demande de 7 échographies abdomino-pelviennes. La recherche a été infructueuse dans 6 cas sur les 7. Le testicule non descendu est un motif fréquent de consultation en pédiatrie et en chirurgie pédiatrique. Son diagnostic repose sur 1'examen clinique au terme duquel 1'examinateur doit pouvoir préciser le caractère palpable ou non du ou des testicules et leur position. Il est rapporté que l'échographie est moins fiable que l'examen clinique dans la détection des testicules non descendus (Le Bartz, Petit, \& Ravasse, 2006). Elle méconnaît le diagnostic de testicule oscillant, elle ne visualise pas les testicules abdominaux et elle ne modifie de ce fait en rien la conduite du traitement chirurgical. La qualité de l'équipement utilisé notamment la fréquence de la sonde d'échographie et la résolution de l'image joue aussi un rôle dans la mise en évidence ou non du testicule non descendu.

En effet plus la fréquence de la sonde superficielle est élevée avec une bonne résolution, plus on a des chances de retrouver le testicule non descendu quand sa topographie est très superficielle. L'échographie cervicale était pathologique dans 6 cas sur 8. Les lésions observées étaient en majorité les adénopathies cervicales (4 cas sur 6). C'est la cause la plus fréquente de la tuméfaction de la région cervicale de l'enfant (Costa De Araujo \& Battisti, 2010). Ces adénopathies étaient une composante cervicale d'adénopathies généralisées ou d'adénopathies localisées exclusivement dans la région cervicale.

La fréquence de l'ETF est relativement faible $(1,6 \%)$ du fait que beaucoup d'anomalies cérébrales sont dépistées en anténatale avec souvent des interruptions thérapeutiques de grossesse pour mauvais pronostic (N'timon, al., 2014). La macrocrânie était principalement le motif de la demande des ETF et ce résultat est superposable à celui d'Adjénou et al (Adjenou, et al., 2012). Elle doit être réalisée en première intention devant cette constatation si les fontanelles ne sont pas encore fermées (Hanquinet, 
Ferey, \& Kalifa, 2009). Elle sera complétée en cas d'atypie par une imagerie en coupe de préférence, l'imagerie par résonance magnétique (IRM).

\section{Conclusion}

Cette étude nous a permis de nous rendre compte que les demandes des échographies pédiatriques sont faibles. Les échographies abdominales étaient les plus demandées avec les affections hépatiques comme principales lésions retrouvées suivi des affections spléniques et rénales. L'échographie cervicale occupait le deuxième rang avec comme principale lésion observée les adénopathies. Bien que de demande relativement faible, l'échographie est d'une aide incontournable dans la prise en charge des affections pédiatriques.

\section{References:}

1. Adjenou K., Amadou A., Adigo A., Agoda-Koussema L., Sonhaye L., Adambounou K., \& al., 2012. ETF et TDM dans le diagnostic des hydrocéphalies chez l'enfant à Lomé. J. Rech. Sci. Univ. Lomé (Togo), 14(2), 39-45.

2. Agoda-Koussema L., Koffi K., Adjenou K. V., Afansounoudji A., Amouzou K., Awobanou K., \& al., 2013. La pratique de la radiologie conventionnelle au Togo: A propos de 580 cas colligés aux $\mathrm{CHU}$ de Lomé. J Afr Imag Med, 5(1), 75-8.

3. Chateil J., Rouby C., Brun M., Labessan C., \& Diard F., 2004. Mesure pratique de l'irradiation en radiopédiatrie : utilisation du produit dose surface en fluorographie numérique et pour les radiographies pulmonaires néonatales. J Radiol, 85, 619-25.

4. Costa De Araujo P., \& Battisti O., 2010. Comment j'explore une masse cervicale de l'enfant. Rev Med Liège, 65(1), 40-45.

5. Delamare, J., 2006. Dictionnaire illustré des termes de médecine. Paris: Maloine. p267.

6. Erdemir A., Kahramaner Z., Cicek E., Turkogl E., Cosar H., Sutcuoglu S., \& al., 2013. Reference ranges for sonographic renal dimensions in preterm infants. Pediatr Radiol, 43, 1475-1484.

7. Hanquinet S., Ferey S., \& Kalifa G., 2009. Imagerie pédiatrique pratique. Elsevier Masson ; 422p.

8. Kouame N., Dieth A. G., Soumma A., Manewa F. S., Ngoan-Domoua A.-M., \& Ngbesso R.-D., 2017. Prise en charge des douleurs abdominales aigues non traumatiques de l'enfant à Abidjan: le couple radiographie standard/échographie est-il encore d'actualité? J Afr Imag Méd, 9(1), 17-20.

9. Labrune P., Trioche-Eberschweiler P., Mollet-Boudjemline A., \& Gajdos V., 2007. Découverte d'une hépatomégalie. Archives de pédiatrie, 14, 573-575. 
10. Le Bartz G., Petit T., \& Ravasse P., 2006. Qu'attendre de l'échographie dans les anomalies de migration testiculaire? Archives de pédiatrie, 13, 426-428.

11. Mabiala-Babela J., Pandzou N., Koutaba, E., Ganga-Zandzou S., \& Sanga P., 2006. Etude rétrospective des urgences chirurgicales viscérales de l'enfant au CHU de Brazzaville (Congo). Méd Trop, 66, 172-176.

12. N'timon B., Amadou A., Aboubakari S. et al., 2014. Depistage échographique des malformations fœtales au Togo. J. Rech. Sci Univ. Lomé(Togo), 2014, Série D, 16(3) : 259-268.

13. Ould Benazzouz Y., Ghannane H., \& Benali S., 2014. Prise en charge de l'hydrocéphalie malformative chez les enfants moins de à propos de 122 patients. Marrakech: Faculté de Médecine et de Pharmacie.

14. Tchaou M., Balde A., Tchangai B., Amadou A., N'timon B., Dagbe M., \& al., 2017. Echographie abdomino-pelvienne pédiatrique en contexte non traumatique au CHU Sylvanus Olympio de Lomé. J Afr Chir Digest, 17(1), 2131-2137. 